\title{
EDITORIAL
}

\section{Treatment of Hypertension in Patients with CAD}

Hence hypertension is the number one risk factor for CVD this situation should manage properly. In this context a scientific statement from the American college of Cardiology, American Heart Association and American Society of Hypertension has been published recently in the JACC . Recommendations are given for the Treatment of Hypertension in patients with Coronary Artery Disease. Following paragraphs have been adopted from this statement.

\section{BPGOAL}

1. The $<140 / 90-\mathrm{mm} \mathrm{Hg}$ BP target is reasonable for the secondary prevention of cardiovascular events in patients with hypertension and CAD .

2. A lower target $\mathrm{BP}(<130 / 80 \mathrm{~mm} \mathrm{Hg})$ may be appropriate in some individuals with CAD, previous MI, stroke or transient ischemic attack, or CAD risk equivalents (carotid artery disease, PAD, abdominal aortic aneurysm)

\section{Patients with CAD and Chronic Stable Angina}

1. Should be treated with a regimen that includes:
a) $\beta$-blocker in patients with a history of prior MI
b) An ACE inhibitor or ARB if there is prior MI, LV systolic dysfunction, diabetes mellitus, or CKD; and
c) A thiazide or thiazide-like diuretic .

2. The combination of a $\beta$-blocker, an ACE inhibitor or ARB, and a thiazide or thiazide-like diuretic should also be considered in the absence of a prior MI, LV systolic dysfunction, diabetes mellitus, or proteinuric CKD.

3. If b-blockers are contraindicated or produce intolerable side effects, a nondihydropyridine CCB (such as diltiazem or verapamil) may be substituted, but not if there is LV dysfunction .

4. If either the angina or the hypertension remains uncontrolled, a long-acting dihydropyridine CCB can be added to the basic regimen of b-blocker, ACE inhibitor, and thiazide or thiazide-like diuretic.

5. There are no special contraindications in hypertensive patients for the use of antiplatelet or anticoagulant drugs, except that in patients with uncontrolled severe hypertension who are taking antiplatelet or anticoagulant drugs, the BP should be lowered without delay to reduce the risk of hemorrhagic stroke .

\section{Patients with ACS and HTN}

1. If there is no contraindication to the use of b-blockers, in patients with ACS, the initial therapy of hypertension should include a short-acting b1selective b-blocker without intrinsic sympathomimetic activity (metoprolol tartrate or bisoprolol). b-Blocker therapy should typically be initiated orally within 24 hours of presentation. For patients with severe hypertension or ongoing ischemia, an intravenous bblocker (esmolol) can be considered .

2. In patients with ACS and hypertension, nitrates should be considered to lower BP or to relieve ongoing ischemia or pulmonary congestion

3. If there is a contraindication to the use of a $\beta$-blocker or intolerable side effects, then a nondihydropyridine CCB such as verapamil or diltiazem may be substituted for patients with ongoing ischemia.

4. An ACE inhibitor or an ARB should be added if the patient has an anterior MI, if hypertension persists, if the patient has evidence of LV dysfunction or HF, or if the patient has diabetes mellitus. For lower risk ACS patients with preserved LV ejection fraction and no diabetes mellitus, ACE inhibitors can be considered a first-line agent for BP control .

5. Aldosterone antagonists are indicated for patients who are already receiving b-blockers and ACE inhibitors after MI and have LV dysfunction and either HF or diabetes mellitus.

6. Loop diuretics are preferred over thiazide and thiazidetype diuretics for patients with ACS who have HF (NYHA class III or IV) or for patients with CKD and an estimated glomerular filtration rate $<30 \mathrm{~mL} / \mathrm{min}$.

For patients with persistent hypertension not controlled with a b-blocker, an ACE inhibitor, and an aldosterone antagonist, a thiazide or thiazide-type diuretic may be added in selected patients for BP control . 
7. The target $\mathrm{BP}$ is $<140 / 90 \mathrm{~mm} \mathrm{Hg}$ in patients with ACS who are hemodynamically stable. A BP target of $<130 /$ $80 \mathrm{~mm} \mathrm{Hg}$ at the time of hospital discharge is a reasonable option.

\section{Patients with HF and HTN}

1. Management of risk factors dyslipidemia, obesity, diabetes mellitus, smoking and dietary sodium and a closely monitored exercise program .

2. Patients should be treated with ACE inhibitors (or ARBs), b-blockers (carvedilol, metoprolol succinate, bisoprolol, or nebivolol), and aldosterone receptor antagonist.

3. Thiazide or thiazide-type diuretics should be used for BP control and to reverse volume overload and associated symptoms.

4. Studies have shown equivalence of benefit of ACE inhibitors and the ARBs candesartan or valsartan in,HF with reduced ejection fraction. Either class of agents is effective in lowering $\mathrm{BP}$

5. The aldosterone receptor antagonists spironolactone and eplerenone have been shown to be beneficial in $\mathrm{HF}$ and should be included in the regimen if there is HF (NYHA class II-IV) with reduced ejection fraction $(<40 \%)$.
6. Hydralazine plus isosorbide dinitrate should be added to the regimen of diuretic, ACE inhibitor or ARB, and b-blocker in African American patients with NYHA class III or IV HF with reduced ejection fraction.

7. In patients who have hypertension and HF with preserved ejection fraction, the recommendations are to control systolic and diastolic hypertension ventricular rate in the presence of atrial fibrillation and pulmonary congestion and peripheral edema .

8. Use of b-adrenergic blocking agents, ACE inhibitors, ARBs, or CCBs in patients with HF with preserved ejection fraction and hypertension may be effective to minimize symptoms of HF .

9. In IHD, the principles of therapy for acute hypertension with pulmonary edema are similar to those for STEMI and NSTEMI, as described above .

\section{Professor Sajal K Banerjee}

Associate Professor Harisul Hoque

Department of Cardiology

BSMMU

Adopted from JACC VOL. 65, NO. 18, 2015 Rosendorff et al. MA Y 12, 2015 : $1998-2038$ Treatment of Hypertension in Patients With CAD 